\title{
Studying the Reversal Mode of the Magnetization Vector Versus Applied Field Angle Using Generalized Magneto-Optical Ellipsometry
}

\author{
M.R. Pufall \\ Department of Physics and Center for Magnetic Recording Research \\ University of California - San Diego, LaJolla, CA 92093 \\ and \\ A. Berger \\ Materials Science Division \\ Argonne National Laboratory, Argonne, IL 60439 \\ The submitted manuscript has been created \\ by the University of Chicago as Operator of \\ Argonne National Laboratory ("Argonne") \\ under Contract No. W-31-109-ENG-38 with \\ the U.S. Department of Energy. The U.S. \\ Government retains for itself, and others act- \\ ing on its behalf, a paid-up, nonexclusive, \\ irrevocable worldwide license in said article \\ to reproduce, prepare derivative works, dis- \\ tribute copies to the public, and perform pub- \\ licly and display publicly, by or on behaif of \\ the Government.
}

Submitted to the 44th Annual Conference on Magnetism and Magnetic Materials, San Jose, CA, Nov. 15-18, 1999

\footnotetext{
Work supported by the U.S. Department of Energy Basic Energy Sciences-Materials Sciences under contract \#W31-109-ENG-38 (at Argonne) and DE-AC03-76SF00098 (at Berkeley) and by the National Science Foundation Contract No. DMR-9805222 (at Berkeley).
} 


\section{DISCLAIMER}

This report was prepared as an account of work sponsored by an agency of the United States Government. Neither the United States Government nor any agency thereof, nor any of their employees, make any warranty, express or implied, or assumes any legal liability or responsibility for the accuracy, completeness, or usefuiness of any information, apparatus, product, or process disclosed, or represents that its use would not infringe privately owned rights. Reference herein to any specific commercial product, process, or service by trade name, trademark, manufacturer, or otherwise does not necessarily constitute or imply its endorsement, recommendation, or favoring by the United States Government or any agency thereof. The views and opinions of authors expressed herein do not necessarily state or reflect those of the United States Government or any agency thereof. 


\section{DISCLAIMER}

Portions of this document may be illegible in electronic image products. Images are produced from the best available original document. 


\title{
27245
}

\section{Studying the Reversal Mode of the Magnetization Vector versus Applied Field Angle using Generalized Magneto-Optical Ellipsometry}

\author{
M. R. Pufall \\ La Jolla, CA 92093 \\ A. Berger \\ Argonne National Laboratory, Argonnne IL
}

Department of Physics and Center for Magnetic Recording Research, University of California, San Diego, 9500 Gilman Dr.,

(August 30, 1999)

We used the technique of vector Generalized Magneto-optical Ellipsometry to study the behavior of the magnetization vector of a 50 Co thin film as a function of external field magnitude and direction. With this method, which determines the both the direction and magnitude of the magnetization, averaged over the $1 \mathrm{~mm}$ incident laser beam, we were able to determine the relative contributions of magnetization rotation and domain formation to the reversal of $M$. The Co sample had a uniaxial in-plane anisotropy. We found that when the angle between the applied field and the easy axis was greater than $\sim 40$ degrees, the reversal occurred primarily by rotation of the magnetization, accompained by a small reduction of the magnitude of $M$. In this angular region, the critical field-the field at which there is a large jump in the angle of $\mathbf{M}$-as a function of applied field angle followed a coherent rotation model. However, at applied field angles less than 40 degrees to the easy axis, we found a larger reduction in $|M|$ occurring before and during the jump in the magnetization angle. The jump also occurred at fields much lower than those predicted by the coherent rotation model, indicating a reversal mode initiated by domain formation. 


\section{INTRODUCTION}

Though a simple experiment conceptually, the hysteresis loop of a sample nonetheless contains a wealth of information. Properly executed and interpreted, the measurement of a hysteresis loop can provide a wide range of information about the sample, from basic parameters such as the remanant magnetization and coercive field, to microstructural properties such as exchange bias, anisotropy axes, the presence of pinning sites, or morphological ripple. The magnetization of a sample may reverse directions in a variety of ways: by rotation of the magnetization direction as a single domain, by the nucleation and growth of reversed domains, or by a combination of both of these processes. Knowledge of this reversal mode is of great importance to the characterization and understanding of novel magnetic materials and structures. So, when studying the mode of magnetization reversal, one must use a method of measuring the hysteresis loop which can determine the magnetization vector-that is, both the direction and magnitude of the magnetization.

Many techniques exist to measure the magnetization vector, utilizing a variety of physical phenomenae. In this report we present the measurement of the magnetization reversal mode of a thin Co film with uniaxial in-plane anisotropy, using the technique of Generalized Magneto-optical Ellipsometry (hereafter termed GME). [1] With this technqiue, we determined both the net magnitude and direction of the magnetization as a function of applied field strength, and as a function of the angle between the applied field and the easy anisotropy axis of the sample, to explore how the reversal mode's character changes with applied field direction.

The GME technique utilizes the three Kerr effects-longitudinal, polar, and transverse-to determine the direction of the magnetization vector. In addition, the same GME measurement returns the optical $(n+i k)$ and magnetooptical $\left(Q_{r}+i Q_{i}\right)$ constants of the sample. Since $Q$ is proportional to $|M|$, the technique can fully determine $\mathbf{M}$, with the magnitude given as a fraction of the saturation magnetization. Many other magneto-optical techniques have been developed to study vector magnetization reversal, such as that of Daboo et. al. [5] Their technique used the longitudinal Kerr effect to determine the in-plane magnetization vector, and used precision rotation stages to rotate the entire sample and field coils by 90 degrees, and so measured two orthogonal components of magnetization. In contrast, GME holds the sample and field coils fixed, and rotates only the input beam's polarization, and the direction of the analyzer.

From the mode of reversal versus applied field direction, one can determine information about the structure of the

sample. For example, by determining the angular behavior of the magnetization versus field, one can determine the 
direction of uniaxial or cubic anisotropy axes. [6] From the magnitude of the magnetization, one sees the degree of domain nucleation, and so determine information about the purity or crystallinity of the sample. Also, one can gain information about the effect of morphological variations in the sample which produce variations in the anisotropy . direction and magnitude, as in the case of magnetization 'ripple.'

\section{EXPERIMENT}

The basic GME setup and measurement procedure has been discussed in detail elsewhere [1], and will be briefly reviewed here. In this study, the sample we measured was a $50 \mathrm{~nm}$ thick Co film sputter-deposited in a magnetic field to give a uniaxial in-plane anisotropy. It was deposited on an $\mathrm{Si}$ substrate, which had a thin oxide layer from atmospheric exposure. The Co film had a $5 \mathrm{~nm}$ sputtered $\mathrm{SiO}_{2}$ capping layer to prevent oxidative degradation of the film. This sample was mounted on a manual rotation stage. The rotation stage had a 0.05 degree accuracy. The external field was applied a pair of air-core electromagnet coils which applied a field oriented in the plane of incidence of the probe beam, with current supplied by a computer-controlled programmable current supply. The incident beam was the $632.8 \mathrm{~nm}$ line from a HeNe laser. The beam first passed through a quarter-wave plate oriented at 45 degrees to its initially linear polarization direction, producing roughly circular polarization. [2] It then passed through a computer-rotatable polarizer, was reflected from the sample through a computer-controlled analyzer, and finally onto a Si detector.

The basic idea of GME is to create an intensity map I(polarizer, analyzer) at a given applied field along the hysteresis cycle. These intensity maps are each then fit to general magneto-optical reflectivity matrices. Reflectivity matrices from a magneto-optical model are then fit to the experimental reflectivity matrices. The model reflection matrices are functions of the parameters of interest-in this case $n, Q$, and magnetization angle. In practice, it is simpler and more accurate to measure a hysteresis at each polarizer, analyzer angle-pair, and then reorganize the data into single field intensity maps.

The measurement procedure is to initially position the sample at some angle relative to the field (The true angle of the anisotropy is initially unknown, but can be determined from the measurement.) The polarizer is initally positioned at an angle several degrees from the p-polarization (polarization in the plane of incidence) direction, and the analyzer several degrees from the orthogonal direction. P-polarization is used because it gives sensitivity to all three components of the magnetization. The hysteresis loop is measured sequentially for many polarizer/analyzer angle pairs around the crossed-polarizers state. This procedure was repeated for many different angles of the sample relative to the applied 
field direction. [3] The data are then reorganized to create an intensity map I(polarizer, analyzer) at a given applied field along the hysteresis cycle. These intensity maps are each then fit to general reflectivity matrices.

No assumptions were made about the sample in determining the reflectivity matrix as a function of applied field, - except the linearity in $M$ of the magneto-optical effects of interest. To model the magneto-optical reflectivity of a multilayer, we used the multilayer matrix formalism developed by Yeh, which was extended to tensor dielectric constants by Visnovsky. [4] Assuming the previosuly measured thicknesses of each layer in the structure, and using the published values of the dielectric constants of $\mathrm{Si}$ and $\mathrm{SiO}_{2}$, we determined the optical and magneto-optical constants of the Co layer, as well as the magnetization angle in the plane. We also measured the out-of-plane vector component and found to be neglible, and so it was neglected in this fit.

\section{RESULTS AND DISCUSSION}

The optical and magneto-optical constants we obtained for Co were $n+i k=2.503+i 4.134$, and $Q_{\text {sat }}=0.02988-$ i0.01220. The error on $n$ was $+/-0.002$, while on $Q$ it was $+/-0.0005$. The index of refraction was essentially constant vs. field. Since $Q$ is proportional to the magnitude of $\mathrm{M}$, we can rewrite $Q(H)=Q_{s a t} M(H)$, and so present magnetization vs. applied field. Figure 1 shows the results of the analysis of GME measurements at two applied field angles, showing the magnetization magnitude and angle vs. applied field strength. In each case there are similar features: a reduction in the magnitude of $M$, and a jump in the magnetization angle, with the reduction in $M$ preceding or coincident with the jump in angle. However, one can also see that the character of the magnetization reversal changes dramatically with applied field angle. When the field is applied near the hard axis (angle $=90^{\circ}$ ), the region of reduced magnetization around the jump in angle is broad and shallow, while nearer the easy axis, the reduced-M region is narrower and deeper. Furthermore, the field at which the magnetization reversal occurs moves monotonically to lower fields with decreasing applied field angle between the applied field and the sample easy axis.

To characterize the changes in the reversal process with sample angle, we fit the magnetization angle vs. field data to a Stoner-Wohlfarth coherent rotation model with the anisotropy as the fit parameter [7] [8]. The results of this fit show that for sample angles (relative to the applied field direction) greater than $\sim 40$ degrees, the effective anisotropy is constant. Consequently, the reversal modes in this angular region are similar, and can be roughly described by a simple coherent rotation model.

However, below this applied field angle, the effective anisotropy begins to decrease with decreasing angle. Figure 2 shows the the critical field-the field at which the magnetization angle jumps abruptly-as a function of applied field 
angle. The field at which the magnetization jump occurs decreases monotonically with decreasing angle between the applied field and easy axis. These values are not well described by a coherent rotation model, which predicts an increasing critical field with decreasing angle. In this region of applied field angle, the reversal mode is dominated by - reversed domain nucleation and growth. Figure 3 shows the minimum magnitude of $M$ achieved at a given applied field angle, as a function of applied field angle. Note that the depth of the minimum deepens as the applied field approaches the easy axis direction.

Finally, we investigated the relationship between the jump in magnetization angle and the onset of domain formation. Figure 4 shows the field at which $M$ just begins to reduce in magnitude, and the critical angular-jump field, versus applied field angle. Also shown on the plot is the field at which the magnetization is a minimum. Note that the magnetization begins to reduce at or just before the angular jump. Also, the magnetization minimum does not occur until just after the critical angular-jump field, indicating that only a fraction of the sample is undergoing the coherent rotation and jump. When the applied field is $>40$ degrees, it takes several Oe beyond the jump field to reverse this small fraction of domains, while around the easy axis, the entire process, both rotation and switching, occurs in a small field range. Figure 3 gives the minimum of $M$ vs. applied field, showing the fraction of the sample involved in the domain formation. The minimum decreases with increasing applied field angle, indicating that there is a small but relatively persistent fraction of the magnetization not undergoing rotation in the large-angle region.

\section{CONCLUSION}

In this report, we have presented a detailed study of the near-DC magnetization reversal mode in a Co thin film with in-plane uniaxial anisotropy, using generalized magneto-optical ellipsometry. We found that the reversal mode changed markedly as a function of the angle of the anisotropy axis relative to the applied field direction. For fields applied $>40$ degrees to the easy axis direction, reversal was primarly by rotation of magnetization, accompanied by a modest reduction in the magnitude of the magnetization. The critical angular-jump fields for applied field angles in this range, as determined from a coherent rotation model for a uniaxial sample, were adequately described by a single anisotropy constant. In contrast, for applied fields $<40$ degrees to the easy axis, the angular rotation of the magnetization was accompanied by a strong dip in the magnitude of the magnetization. In addition, the critcal angular-jump fields in this region were much lower than those expected from a coherent rotation model, due to the nucleation of domains. 
[1] Berger, A. and Pufall, M.R. "Generalized Magneto-Optical Ellipsometry", Appl. Phys. Lett. 71, 965 (1997).

[2] This is only done to make the intensity out of the polarizer and subsequently incident on the sample roughly constant with polarizer angle, for experimental convenience, but is irrelevent to the magneto-optics.

[3] The magneto-optical model we used is linear in $M$, so magneto-optical effects that are $O\left(M^{2}\right)$, should be removed from the data. We accomplished this by making the ansatz that $M(H)^{+}$-that is, the magnetization at field $H$, with the field increasing-and $-M(-H)^{-}$, the negative of the magnetization at $-H$, with the field decreasing, are the same. Factors $\mathrm{O}\left(\mathrm{M}^{2}\right)$ do not reverse sign, leaving a loop linear in $M$ (and very small higher-order odd terms).

[4] Visnovsky, S., "Magneto-Optical Ellipsometry", Czech. J. Phys. B 36, 625 (1986.

[5] Daboo, C., Bland, J. A. C.,et. al., "Vectorial Magnetometry with the magneto-optic Kerr effect applied to Co/Cu/Co trilayer structures," Phys. Rev. B 47, 11852 (1993).

[6] Daboo, C., Hicken, R.J., et. al., "Anisotropy and orientational dependence of magnetization reversal processes in epitaxial ferromagnetic thin films," Phys. Rev. B 51, 15964 (1995).

[7] Stoner, E.C. and Wohlfarth, E.P., "A Mechanism of Magnetic Hysteresis in Heterogenous Alloys," Philos. Trans. R. Soc. A 240, 599 (1948).

[8] Obviously since domains are forming, this model is not strictly applicable, but the degree and manner of deviations from the expected values are informative 


\section{FIGURE CAPTIONS}

FIG. 1. Plot of magnitude and direction of $\mathbf{M}$ around reversal

FIG. 2. Critical field vs. applied field angle

FIG. 3. Minimum $|M|$ vs. applied field angle

FIG. 4. Plot of domain onset, angular jump, and minimum $|M|$ fields vs. applied field angle 


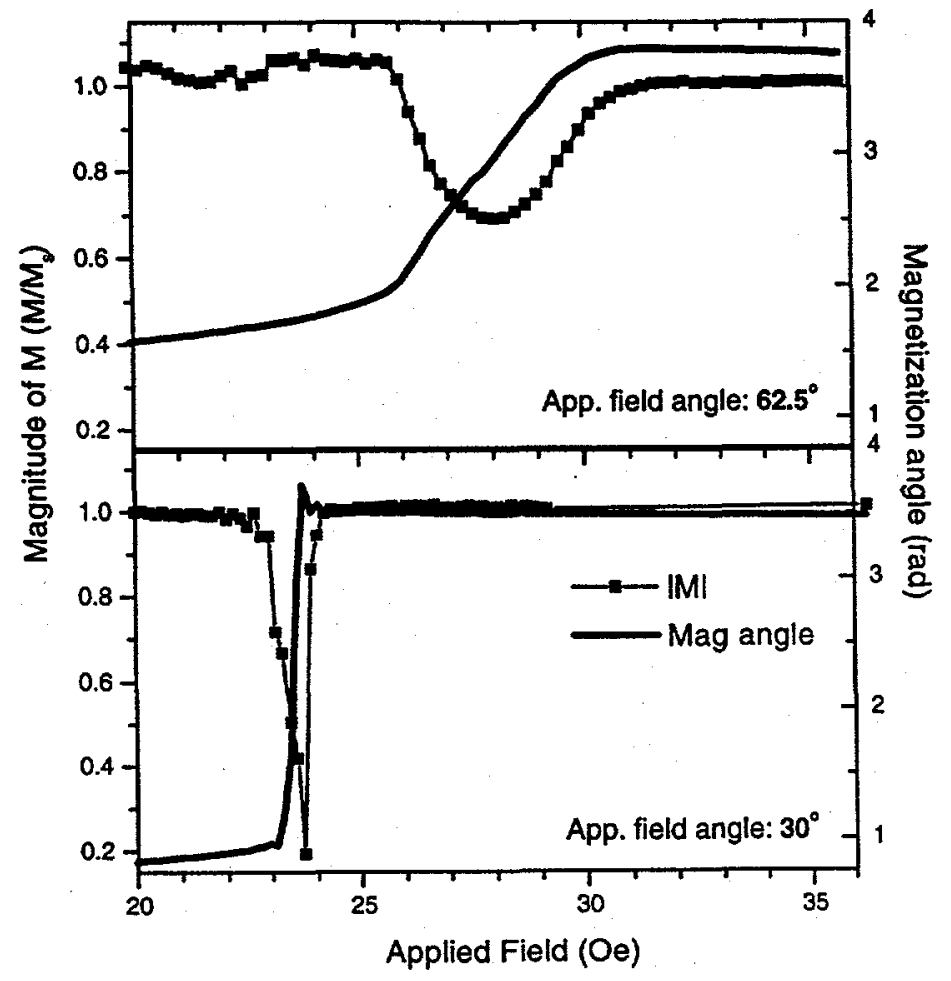

Figure 1: Plot of magnitude and direction of $\mathbf{M}$ around reversal 


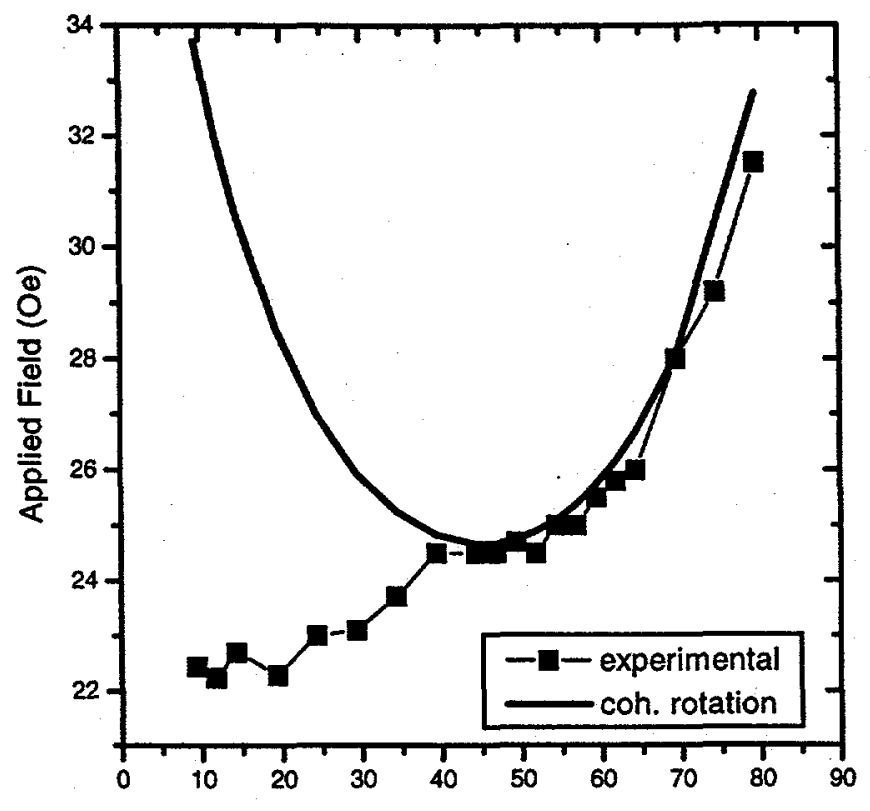

Field angle (rel. to easy axis)

Figure 2: Critical field vs. applied field angle 


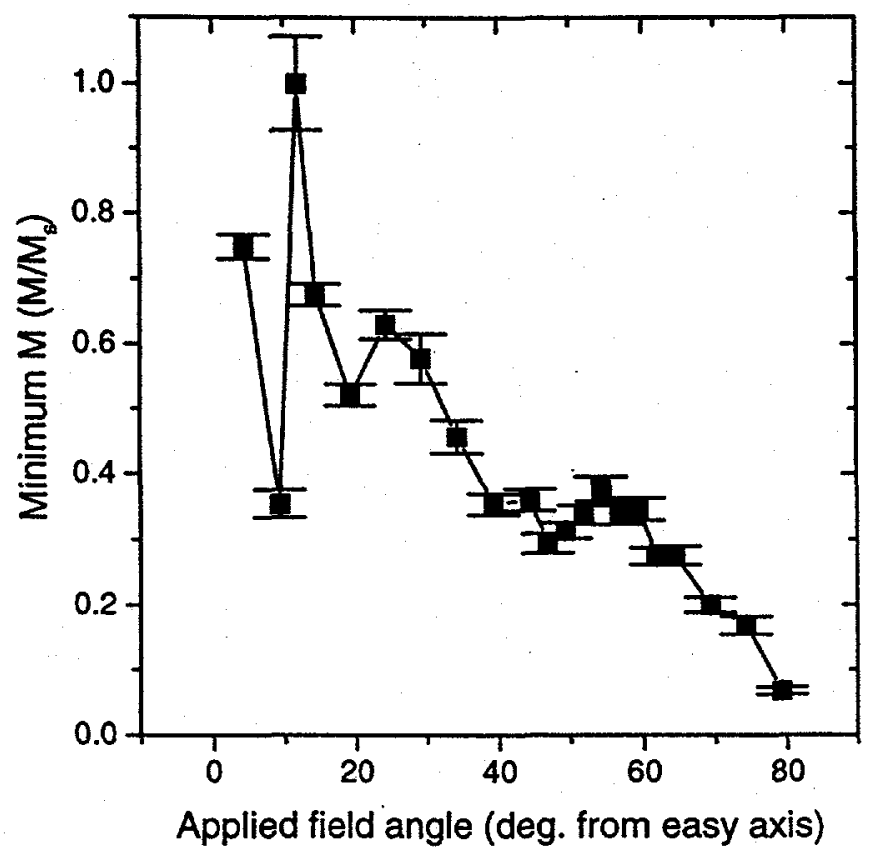

Figure 3: Minimum IMI vs. Applied Field Angle 


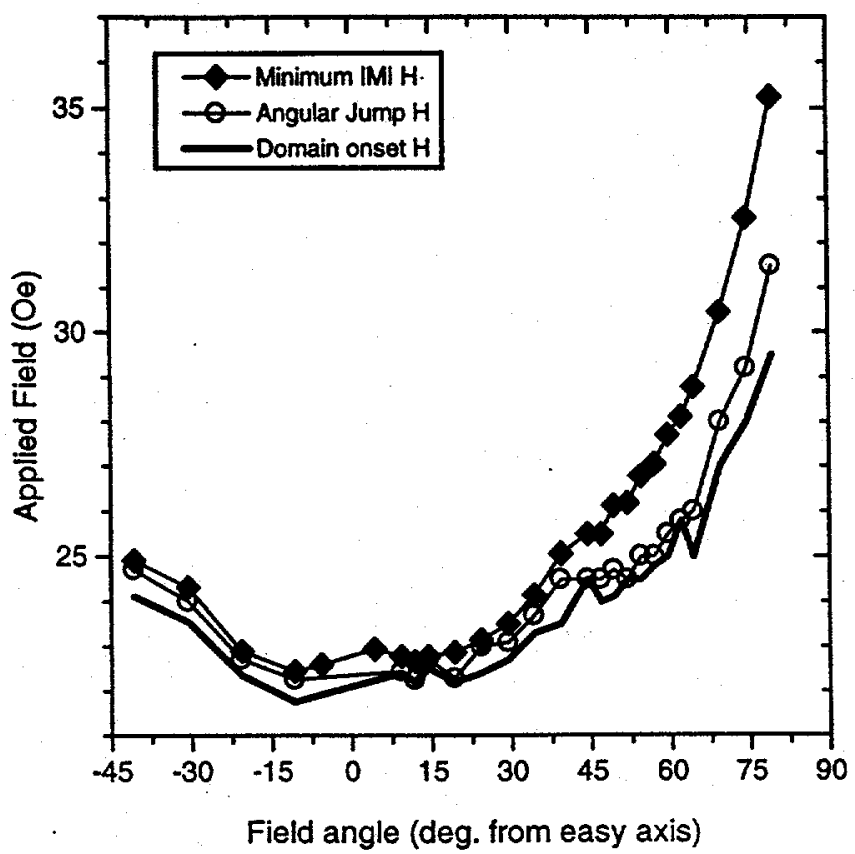

Figure 4: Plot of domain onset, angular jump, and minimum IMI fields vs. applied field angle 